nalysetools «Similarweb» (2019) erhoben, wie viele monatliche Seitenzugriffe sie verzeichnet und aus welchen Ländern diese stammen. Weiter mitaufgezeichnet wurden die Präsenz bzw. die Community-Grössen auf den Social-Media-Plattformen Facebook, Twitter und YouTube. Dies mit dem Ziel, ein breites Bild von den Online-Aktivitäten der Nachrichtensites zu erfassen. Der daraus resultierende Datenpool war grundlegend für alle weiteren methodischen Schritte.

\title{
VI.2 Qualitative Analyse alternativer Newswebsites
}

Nachdem im vorangegangenen ersten methodischen Schritt das Ziel darin bestand, möglichst viele alternative Nachrichtenwebsites zu erheben, um einen breiten Überblick über den derzeitigen Bestand in den drei Untersuchungsländern zu geben, soll im nächsten Schritt ein detaillierter, interpretativer Blick erfolgen. Die Webauftritte der alternativen Nachrichtenmedien werden entsprechend der Forschungsfrage 2 dahingehend untersucht, nach welchen Kriterien sich diese ihrem Selbstverständnis zufolge definieren und kategorisch unterscheiden lassen. Da es sich hierbei um eine offene, explorative Forschungsfrage handelt, mit dem Ziel, eine theoretische Weiterentwicklung dahingehend $\mathrm{zu}$ schaffen, wie sich diese Art von Gegenöffentlichkeiten einordnen lassen, fiel die Wahl auf ein qualitatives Forschungsdesign auf Grundlage der Grounded-Theory-Methodologie. Der Zugang ist phänomenologisch, weshalb das primäre Ziel darin besteht, den subjektiv gemeinten Sinn zu identifizieren, um somit Phänomene erklären zu können. Eine normative Wertung ist in diesem methodischen Schritt zu vermeiden.

Die Methodologie der Grounded Theory (oder: der «gegenstandsbezogenen Theoriebildung») hat eine lange Tradition und im Laufe der letzten Jahrzehnte unterschiedliche Ansätze hervorgebracht. Entwickelt von den amerikanischen Soziologen Barney Glaser und Anselm Strauss (1967) in den 1950er- und 6oer-Jahren besteht das Ziel dieses Forschungsansatzes darin, während des Forschungsprozesses Empirie und Theoriebildung stark miteinander zu verzahnen. Ein Aufstellen von Hypothesen im Vorfeld würde folglich genau dieser Logik widersprechen, da eine höchstmögliche Offenheit bei der Konzeptbildung (im Sinne von Codes und Konstrukten) bewahrt werden soll, die zudem durch zirkuläre Datenerhebungs- und Auswertungsprozesse immer weiterentwickelt werden (Przyborski \& Wohlrab-Sahr, 2014, S. 191ff.). Grundsätzlich wird unter der Grounded Theory eine Theorie verstanden, die induktiv aus der Erforschung des Phänomens selbst entwickelt wird. Sie basiert auf dem Prozess systematischer Datensammlung, Datenauswertung und Theoriebildung. Diese Schritte werden indes nicht nacheinander durchgeführt, sondern stehen im Sinne eines Kreislaufes in wechselseitigem Bezug zueinander (Strauss \& Corbin, 1990, S. 23). Wesentlicher Grund für die Entwicklung der Theorie war das Bestreben der beiden Autoren, die Kluft zwi- 
schen empirischer Forschung und formaler Theorie zu verringern. Zudem sollte vermieden werden, qualitative Forschung als blosse «Vorarbeit» für quantitative Verfahren zu betrachten (Przyborski \& Wohlrab-Sahr, 2014, S. 195).

Obwohl die beiden «Gründer» Glaser und Strauss gemeinsam mit der Entwicklung der Grounded Theory Methodology gestartet haben, gibt es mittlerweile zwei Stränge, die sich teilweise voneinander unterscheiden. So kooperierte Anselm Strauss mit Juliet Corbin, mit der er 1990 gemeinsam das Buch «Basics of Qualitative Research» publizierte (Strauss \& Corbin, 1990), worauf eine Art Gegenpublikation von Glaser (1992) folgte. Die vorliegende Arbeit schliesst sich vorwiegend den Ausführungen von Strauss und Corbin an, da der forschungslogische Ansatz zumindest für diese Studie plausibler und adäquater erscheint, beispielsweise aufgrund der von den Autoren diskutierten Kontroverse hinsichtlich des Begriffs der «Induktion»: Grundgedanke des induktiven Vorgehens bei Glaser und Strauss ist es, dass Theorien und Konzepte aus den Daten selbst emergieren. Theoretisches Vorwissen sei demnach nicht notwendig und auch gar nicht zielführend. Die ersten Werke empfehlen ein gänzliches «Ignorieren» theoretischer Arbeiten zum Themenbereich, auch um den offenen Blick auf die Daten nicht zu beeinflussen. Während Glaser weiterhin diese Prämisse vertritt, hat sich Strauss (gemeinsam mit Corbin) nach und nach von diesem strikten Gedanken gelöst. Erstens wurde der Ansatz als «überspitzt» formuliert betrachtet und zweitens äusserte sich Strauss in weiterer Folge sogar positiv hinsichtlich des Vorhandenseins von Kontextwissen und Kenntnis der einschlägigen Fachliteratur schon vor bzw. während der Datensammlung, -auswertung und Theoriebildung (Przyborski \& Wohlrab-Sahr, 2014, S. 196; Strauss \& Corbin, 1990, S. 42; 48-56). Im Rahmen der vorliegenden Studie war es wesentlich, diese Diskussion zu reflektieren. Aus forschungspragmatischen Gründen konnte auf die vorangehende Lektüre einschlägiger Literatur nicht verzichtet werden, zumal ein Überblick über den aktuellen Stand der Forschung unumgänglich ist, um bisherige Ansätze und Forschungsdesiderate zu identifizieren. Ebenso besitzt jede_r Forscher_in einen biographischen wie auch professionellen Hintergrund. Die Annahme, man könne ohne theoretische Vorkenntnisse in die Daten eintauchen, erscheint unrealistisch. Dennoch wurde darauf geachtet, sich eine grösstmögliche Offenheit gegenüber dem Phänomen und den Daten zu bewahren, indem vorangehende Literaturrecherchen auf das Nötigste beschränkt wurden. ${ }^{2}$

Es gibt fünf Grundprinzipien, die bei der Anwendung der Grounded Theory Methodology beachtet werden müssen. Erstens folgt die Datenerhebung der Logik des Theoretical Samplings. Dies bedeutet, dass Dokumente zur Weiterentwicklung der theoretischen Konzepte so lange erhoben werden, bis eine «theoretische

2 Aus diesem Grund wurde der theoretische Teil dieser Arbeit erst nach Abschluss der empirischen Forschung systematisch aufgearbeitet und verschriftlicht. 
Sättigung» erreicht ist. Daten werden demnach nicht nach vorab festgelegten Kriterien, sondern in Hinblick auf die Theorieentwicklung abzielend gesammelt, z. B. in Form von kontrastierenden Dokumenten. Erst wenn die Theorie nicht durch zusätzliches Datenmaterial fortentwickelt werden kann, ist das Sampling abgeschlossen. Zweitens erfolgt das Kodieren theorieorientiert, d. h. der Kodierprozess verfolgt das Ziel der Theoriegewinnung, die auch während des Prozesses am Material ständig überprüft werden muss. Drittens beruht die Datenauswertung auf dem Prinzip des ständigen Vergleichens, wonach bereits gebildete Konzepte und Kategorien im Forschungsprozess permanent gegenübergestellt werden, um diese zu schärfen. Viertens sieht die Methodologie vor, während der Auswertung theoretische Memos zu verfassen. Dabei handelt es sich nicht um Konzepte oder Codes, vielmehr rekurrieren sie auf diese, weisen auf Lücken in den theoretischen Überlegungen hin, oder deuten beispielsweise auf weitere Datenerhebungen hin. Fünftens wird auf die Relationierung von Datenerhebung, Kodieren und Memoschreiben im Forschungsprozess hingewiesen. Der Prozess ist folglich nicht linear zu betrachten, vielmehr lebt er von einer wechselseitigen Verzahnung der verschiedenen Arbeitsschritte (Przyborski \& Wohlrab-Sahr, 2014, S. 199-209).

Im Zentrum der Grounded-Theory-Methodologie steht das Erstellen von Konzepten und Kategorien - das Kodieren. Dabei können drei Kodierschritte voneinander unterschieden werden: (1) Offenes Kodieren; (2) Axiales Kodieren; (3) Selektives Kodieren. Der erste Analyseschritt (1) dient dem «Aufbrechen» der Daten. Hierbei wird das Datenmaterial in Form von Texten, Bildern oder Ähnlichem sequenziell analysiert und es werden erste Konzepte entwickelt. Diese Konzepte können als erste Blöcke für die Theoriebildung betrachtet werden, sind allerdings für tiefergehende Interpretationen noch offen. In einem weiteren Schritt können sie in erste Kategorien gruppiert werden, die als identifizierte Phänomene in den Daten beschrieben werden können. Diese Kategorien werden wiederum durch Subkategorien näher beschrieben, aber auch anhand unterschiedlicher «Eigenschaften» oder «Dimensionen» konkretisiert (z. B. kann eine (fiktive) Kategorie «Politik» die Eigenschaft «politische Einstellung» implizieren, die anhand einer Links- vs. Rechtsorientierung dimensioniert wird) (Strauss \& Corbin, 1990, S. 6174). Nachdem erste Konzepte und Kategorien erstellt wurden, müssen diese im zweiten Analyseschritt, dem axialen Kodieren (2), neu miteinander in Beziehung gesetzt werden. Ziel ist es, Kategorien oder Phänomene aus dem Datenmaterial $\mathrm{zu}$ extrahieren, die relevant für die Theoriebildung sein könnten. Wesentliche Kategorien sollten entsprechend genauer ausgearbeitet werden, mit dem Ziel, eine (oder mehrere) Schlüsselkategorie(n) zu identifizieren, die gleichzeitig den Kern der Theorie widerspiegeln soll(en). Das In-Beziehung-Setzen von Kategorien kann mithilfe des von Strauss und Corbin empfohlenen Kodierparadigmas vorgenommen werden. Dabei wird eine Kategorie bzw. ein Phänomen durch folgende Fragen mit anderen (Sub-)Kategorien in Relation gesetzt: Was ist das Phänomen 
(z. B. «Medienkritik»)? Was sind ursächliche Bedingungen für das Phänomen (z. B. «Unzufriedenheit mit Mainstreammedien»)? Welche intervenierenden Bedingungen gibt es (z. B. «Wandel des Mediensystem»)? Was ist der Kontext (z. B. «Journalismusverständnis»)? Welche Strategien gibt es (z. B. «Oppositionelle Rhetorik»)? Und schliesslich: Was sind die Konsequenzen (z. B. «Aufruf zum Protest») (Strauss \& Corbin, 1990, S. 96-115). Der dritte Analyseschritt des selektiven Kodierens (3) beschäftigt sich nun abschliessend mit den im vorangehenden Schritt identifizierten Schlüsselkategorien, anhand derer die Theorie herausgearbeitet werden kann und in die alle weiteren Kategorien integriert werden können. Dabei werden nur mehr die für die Theorie relevanten Kategorien betrachtet und miteinander in Bezug gesetzt (Strauss \& Corbin, 1990, S. 116-134).

Der Ansatz der Grounded Theory eignet sich für die Bearbeitung respektive Beantwortung der zweiten Forschungsfrage deswegen besonders gut, um das Phänomen «alternative Nachrichtenmedien» explorativ zu erforschen. Der phänomenologische Zugang ermöglicht es, das Selbstverständnis der Untersuchungsobjekte aus deren Sichtweise zu identifizieren. Von einer normativen Betrachtung wird bewusst Abstand genommen, um erstens eine intersubjektive Nachvollziehbarkeit zu gewährleisten. Zweitens könnte man Gefahr laufen, den Untersuchungsgegenstand in «gute» versus «schlechte» Nachrichtenmedien zu unterscheiden bzw. diese zu bewerten. Dies soll nicht Zweck dieser Analyse sein. Weiter zielt die Grounded Theory auf die Theorieentwicklung ab. Auch hinsichtlich dieses Punktes überzeugt der methodologische Zugang zur Beantwortung der Forschungsfrage. Schliesslich soll einerseits der Begriff «alternative Nachrichtenmedien» eindeutig definiert und weiter das Phänomen differenzierter betrachtet werden, indem unterschiedliche Typen von Alternativmedien herausgearbeitet werden. In diesem Sinne kann von einer theoretischen Weiterentwicklung gesprochen werden.

Für die qualitativen Auswertungen wurde unterstützend das Tool MAXQDA herangezogen. Über das «Web Collector» Add-on des Browsers Google Chrome konnten die zu analysierenden Websites statisch abgespeichert und direkt in die Analysesoftware importiert werden. Somit konnte der Kodierprozess übersichtlicher gestaltet und vereinfacht werden.

\section{VI.3 Datenerhebung auf Twitter}

Eines der wesentlichen Ziele dieser Forschung besteht darin, die Netzwerke alternativer Nachrichtenmedien zu untersuchen. Um einen strukturellen Blick auf die Relationen von und gegenüber alternativen Nachrichtenmedien zu erhalten, können (quantitative) Netzwerkanalysen durchgeführt werden. Der quantitative Ansatz hilft dabei, einen ersten, deskriptiven Überblick über die Netzwerk- 